\title{
Objective compressive quantum process tomography
}

\author{
Y. S. Teo $\odot,{ }^{1, *}$ G. I. Struchalin $\odot,{ }^{2, \dagger}$ E. V. Kovlakov, ${ }^{2}$ D. Ahn,,${ }^{1}$ H. Jeong, ${ }^{1}$ S. S. Straupe, ${ }^{2}$ \\ S. P. Kulik, ${ }^{2}$ G. Leuchs, ${ }^{3,4}$ and L. L. Sánchez-Soto ${ }^{3,5}$ \\ ${ }^{1}$ Department of Physics and Astronomy, Seoul National University, 08826 Seoul, Korea \\ ${ }^{2}$ Quantum Technologies Centre, and Faculty of Physics, Moscow State University, 119991 Moscow, Russia \\ ${ }^{3}$ Max-Planck-Institut für die Physik des Lichts, Staudtstraße 2, 91058 Erlangen, Germany \\ ${ }^{4}$ Institute of Applied Physics, Russian Academy of Sciences, 603950 Nizhny Novgorod, Russia \\ ${ }^{5}$ Departamento de Óptica, Facultad de Física, Universidad Complutense, 28040 Madrid, Spain
}

(Received 12 December 2019; accepted 29 January 2020; published 26 February 2020)

\begin{abstract}
We present a compressive quantum process tomography scheme that fully characterizes any rank-deficient completely positive process with no spurious a priori information. It uses randomly chosen input states and adaptive output von Neumann measurements. Both entangled and tensor-product configurations are flexibly employable in our scheme, the latter of which are especially compatible with many-body quantum computing. Two main features of this scheme are the certification protocol that verifies whether the accumulated data uniquely characterize the quantum process and a compressive reconstruction method for the output states. We emulate multipartite scenarios with high-order transverse modes and optical fibers to demonstrate that, in terms of measurement resources, our assumption-free compressive strategy can reconstruct quantum processes almost equally efficiently using all types of input states and basis measurements.
\end{abstract}

DOI: 10.1103/PhysRevA.101.022334

\section{INTRODUCTION}

Quantum computers and devices [1-3] employ logic gates [4-8] to carry out computations using $d$-dimensional many-body systems, such as qubit ensembles. The quality of computed results hinges on the reliability of tomographic certifications of these gates, each of which is a quantum process $\Phi$ represented by a positive Choi-Jamiołkowski operator $\rho_{\Phi}[9,10]$, naively requiring $O\left(d^{4}\right)$ informationally complete (IC) measurements [11-14] that are too resource intensive to be implemented for large $d$. Ancilla- [15-18] and error-correction-based [19-22] quantum process tomography (QPT) were introduced to circumvent this problem. For highly specific property prediction tasks, probing selected elements of $\rho_{\Phi}$ is another option [23-28]. One attempt to directly reduce the measurement cost of QPT with non-IC measurements and entropy methods was reported [29]. Simultaneously, the method of compressed sensing [30-36] was applied to QPT [37-39] to reconstruct low-rank or sparse processes with a small set of specialized compressive measurements. However, this concept only works under the assumption that $\rho_{\Phi}$ should either possess a rank no larger than a known integer $r$ or be sparse in some known basis and sparsity, all of which demand reliable evidence. So, target guesses are needed to validate all reconstructions.

In this article, we implement a compressive statereconstruction-assisted quantum process tomography (ACTQPT) scheme that requires no a priori rank or sparsity knowledge, or any other precarious assumptions about $\Phi$.

\footnotetext{
*ys_teo@snu.ac.kr

${ }^{\dagger}$ struchalin.gleb@physics.msu.ru
}

The standard ancilla-free framework shall be considered here, which consists of input states $\left(\rho_{\mathrm{IN}}\right)$ and output-state von Neumann basis measurements that can be feasibly implemented in practice. Our scheme comprises an adaptive compressive (state) tomography (ACT) protocol $[40,41]$ that reconstructs the unknown output states $\left(\rho_{\text {OUT }}=\Phi\left[\rho_{\mathrm{IN}}\right]\right)$ from adaptively chosen bases, and an informational completeness certification that determines whether the process estimator $\widehat{\rho}_{\Phi}$ (distinguished with a hat from the true process operator $\rho_{\Phi}$ ) is uniquely characterized by the accumulated data set or not. This can be achieved with semidefinite programs $[42,43]$. If $\widehat{\rho}_{\Phi}$ is not unique, the scheme is repeated with different linearly independent input states until $\widehat{\rho}_{\Phi}$ is unique. We also develop a product ACTQPT scheme (PACTQPT) that adopts product input states and basis measurements suitable for realistic many-body implementations that avoid sophisticated entanglement manipulation and control. We experimentally test (P)ACTQPT in an emulated many-body setting by encoding high-dimensional states in transverse modes of light with Hermite-Gaussian beams and simulating multiqubit processes with multimode optical fibers. For all tested processes, both ACTQPT and PACTQPT perform comparably and are highly compressive relative to $O\left(d^{4}\right)$.

\section{COMPRESSIVE QUANTUM PROCESS TOMOGRAPHY}

\section{A. Theoretical background}

ACTQPT is an iterative scheme that completely and unambiguously characterizes any unknown rank- $r \rho_{\Phi}$ based solely on data acquired from measuring output states $\left\{\rho_{\text {out }}^{(j)}\right\}$ as a consequence of the unknown process $\Phi$, and nothing else. For this purpose, the scheme is armed with two important components (see Fig. 1). The first component is the ACT 
(a)

\section{$j$ th step of ACTQPT on $\rho_{\Phi}$}

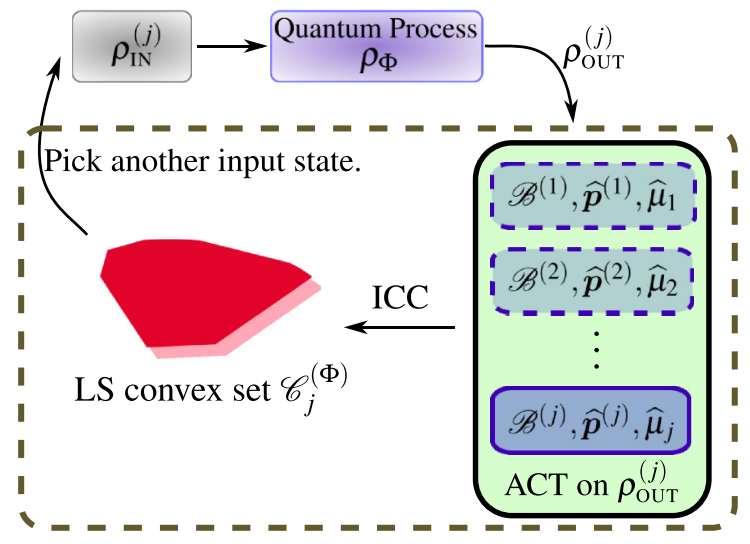

(b)

$k$ th step of ACT on $\rho_{\text {OUT }}^{(j)}$

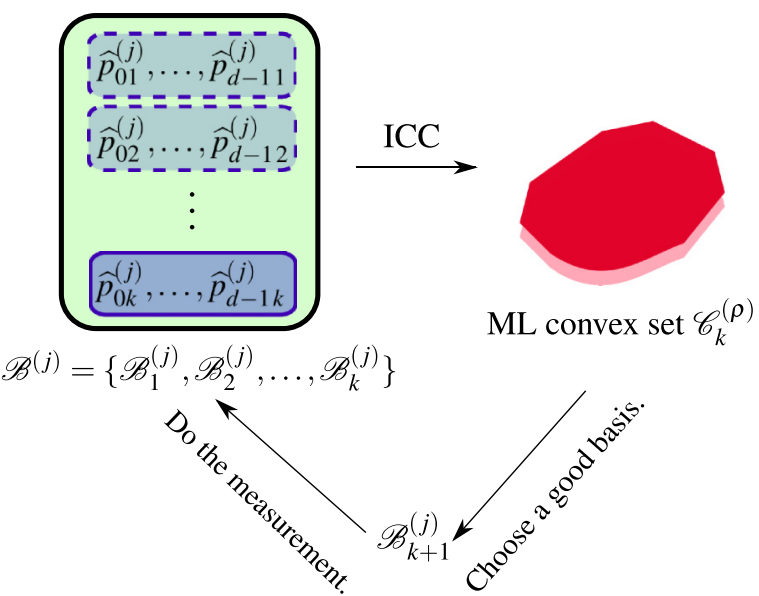

FIG. 1. (a) Flowchart of ACTQPT. An input state $\rho_{\mathrm{IN}}$ through the unknown $\Phi$ leads to an unknown output state $\rho_{\text {OUT }}$ that is compressively characterized with ACT at every step. The accumulated measurement bases sequences $\left\{\mathscr{B}^{(j)}\right\}$, ML probabilities $\left\{\widehat{\boldsymbol{p}}^{(j)}\right\}$, and estimated $\left\{\widehat{\mu}_{j}\right\}$ are analyzed in ICC to deterministically decide if the associated LS convex set $\mathscr{C}^{(\Phi)}$ is singleton or not. If not, ACTQPT picks a new input state for more data collection. ACT is a subroutine run for each $\rho_{\text {OUT }}^{(j)}$, which itself requires iterations. A schematic of the $k$ th ACT iteration is shown in (b). It first performs ICC with the accumulated basis data set to determine whether or not the ML convex set $\mathscr{C}^{(\rho)}$ is singleton. If not, the next basis measurement is chosen as the eigenbasis of a minENT estimator.

scheme $[40,41]$ that chooses a compressive sequence of optimal von Neumann measurements to efficiently characterize every output state. In every step, it first certifies if the accumulated data uniquely characterize, say, $\rho_{\text {OUT }}$ after feeding $\rho_{\mathrm{IN}}$ to $\Phi$. This informational completeness certification (ICC) analyzes the convex space $\mathscr{C}^{(\rho)}$ of quantum states that give the same maximum-likelihood (ML) [44-47] probabilities according to the accumulated data set in order to compute an indicator $s_{\mathrm{CVX}}^{(\rho)}$. If $s_{\mathrm{CVX}}^{(\rho)}=0$, then $\mathscr{C}^{(\rho)}$ is a singleton, a set containing one operator, and ACT terminates. Otherwise, the next optimal measurement is chosen as the eigenbasis of the minimum von Neumann-entropy (minENT) estimator over $\mathscr{C}^{(\rho)}$. This generally leads to very efficient ACT convergences $[41,48,49]$ as a rank-reducing guiding principle.

Open-system quantum processes with losses are usually not trace-preserving (TP), so that $\operatorname{tr}\left\{\rho_{\text {OUT }}\right\} \leqslant 1$. After analyzing $j$ output states, their relative trace values $\left\{\mu_{j^{\prime}}=\right.$ $\left.\operatorname{tr}\left\{\rho_{\text {OUT }}^{\left(j^{\prime}\right)}\right\}\right\}_{j^{\prime}=1}^{j}$ can be estimated (up to a common multiplicative factor), for example, by normalizing the measured counts by their total sum for all the different output states, provided the count rate is sufficiently high and each measurement is conducted under a fixed common duration.

We now discuss the second crucial component, which is an ICC over the convex space $\mathscr{C}^{(\Phi)}$ of processes that are consistent with the set of ML basis probabilities $\left\{\widehat{\boldsymbol{p}}^{\left(j^{\prime}\right)}\right\}$ and estimated $\left\{\widehat{\mu}_{j^{\prime}}\right\}$ derived from all ACT runs so far. By the $j$ th iteration, the operators in $\mathscr{C}_{j}^{(\Phi)}$ are those that give the same least-square (LS) value

$$
\mathscr{D}_{\min }=\min _{\widehat{\Phi}}\left\{\sum_{j^{\prime}=1}^{j} \sum_{k^{\prime}=1}^{K_{j^{\prime}}} \sum_{l^{\prime}=0}^{d-1}\left(\left\langle b_{l^{\prime} k^{\prime}}^{\left(j^{\prime}\right)}\left|\widehat{\Phi}\left[\rho_{\mathrm{IN}}^{\left(j^{\prime}\right)}\right]\right| b_{l^{\prime} k^{\prime}}^{\left(j^{\prime}\right)}\right\rangle-\widehat{\mu}_{j^{\prime}} \widehat{p}_{l^{\prime} k^{\prime}}^{\left(j^{\prime}\right)}\right)^{2}\right\}
$$

where all $K_{j}$ IC ACT measured bases in the set $\mathscr{B}^{(j)}=$ $\left\{\mathscr{B}_{1}^{(j)}, \ldots, \mathscr{B}_{K_{j}}^{(j)}\right\}$ form a sequence of optimally chosen bases $\mathscr{B}_{k}^{(j)}=\left\{\left|b_{l^{\prime} k}^{(j)}\right\rangle\left\langle b_{l^{\prime} k}^{(j)}\right|\right\}_{l^{\prime}=0}^{d-1}$ for $1 \leqslant k \leqslant K_{j}$. The answer to ICC is a uniqueness indicator $s_{\mathrm{CVX}}^{(\Phi)}$, from which we may conclude that $\widehat{\rho}_{\Phi}$ is unique if $s_{\mathrm{CVX}}^{(\Phi)}=0$ and only then. Otherwise, ACTQPT picks another input state randomly, and the procedures of ACT and ICC over $\mathscr{C}^{(\Phi)}$ are repeated until $s_{\mathrm{CVX}}^{(\Phi)}=0$.

The validity of ICC that systematically certifies reconstruction uniqueness with data relies on the fact that both $\mathscr{C}^{(\Phi)}$ and $\mathscr{C}^{(\rho)}$ are convex sets. We briefly present the mechanism of ICC on a generic data convex set $\mathscr{C}^{(X)}$ of bounded Hermitian operators $X$. We start with a simple linear function $f=\operatorname{tr}\{X Z\}$, where $Z$ is a fixed Hermitian operator. In our context, $X$ and $Z$ are either states or process operators. As $\mathscr{C}^{(X)}$ is convex, minimizing and maximizing $f$ over $\mathscr{C}^{(X)}$ gives unique boundary answers, which we hereby denote by $f_{\min }$ and $f_{\max }$, respectively. It remains to show that if $s_{\mathrm{CVx}} \propto$ $f_{\max }-f_{\min }$, then (i) $s_{\mathrm{CVx}}$ is nonincreasing with decreasing volume size $s$ of $\mathscr{C}^{(X)}$ for noiseless data and (ii) $s_{\mathrm{CvX}}=$ $0 \leftrightarrow s=0$ (singleton condition) with any data. For (i), we observe that, as noiseless data accumulate, and hence more distinct linear physical-probability constraints are imposed on $X, f_{\max }$ decreases and $f_{\min }$ increases progressively owing to the linearity of $f$. It is clear that $s_{\mathrm{CVX}}$ also decreases with $s$. Property (ii) follows immediately by noticing that as long as $\mathscr{C}^{(X)}$ is convex and $f$ has no ill-behaved plateau structures (guaranteed by a randomly chosen full-rank $Z$ ), then $s=0$ necessarily implies a singleton $\mathscr{C}^{(X)}$. Whether this singleton set contains the true $X$ or another operator close to it depends on whether noise is present in the data. The optimization of $f$ over $\mathscr{C}$ is, in fact, a semidefinite program (SDP) $[42,43]$. 
ACTQPT is the whole iterative package \{entangled $\rho_{\mathrm{IN}} \mathrm{S}$, ACT, ICC over $\left.\mathscr{C}^{(\Phi)}\right\}$. On the other hand, the compressive measurement sequences obtained from ACT typically constitute entangled bases and are difficult to implement without sophisticated global entangling operations. Thus a much more attractive alternative is to enforce a tensor-product local structure on all measurement bases, which turns ACT into its product counterpart (PACT) that is experiment friendly. This gives rise to another scheme that is much more suitable for many-body systems and quantum devices, namely the product version of ACTQPT (PACTQPT) that requires only subsystem manipulations: $\left\{\right.$ product $\rho_{\mathrm{IN}}$ s, PACT, ICC over $\mathscr{C}^{(\Phi)}$ \}.

\section{B. Minimum-entropy principle}

We shall reiterate minENT procedure for ACT (found in $[40,41])$ in this section. We recall the fundamental fact that minimizing a concave function over convex spaces is generally not a convex problem. This results in the existence of nonunique optimal solutions to choose from the optimization. Semidefinite programs are therefore incompatible with such a problem. Nevertheless, we construct an equivalent and simple barrier method to minimize the von Neumann entropy $S\left(\rho^{\prime}\right)=-\operatorname{tr}\left\{\rho^{\prime} \ln \rho^{\prime}\right\}$ over the ML convex set $\mathscr{C}^{(\rho)}$.

Following [45], we first consider the Lagrange function $\mathscr{D}=-\lambda S+\ln L$ that is a sum of $S$ and the $\log$-likelihood $\ln L$ weighted by a small positive parameter $\lambda \ll 1$, where the $\rho^{\prime}$ dependence is dropped from all functions for notational convenience. We also note that, for any non-IC data set $\mathbb{D}$, the corresponding $L$ (or $\ln L$ ) possesses a plateau structure over the quantum domain $\mathscr{C}^{(\rho)}$. A perfectly accurate minENT state estimator that both minimizes $S$ and remains in $\mathscr{C}^{(\rho)}$, the subspace of quantum states that give the same maximal $L$ value, therefore corresponds to a $\lambda$ that is infinitesimal. As this is never feasible in practice, we approximate this situation with a small finite $\lambda$ such that both conditions are satisfied with a finite precision.

Numerically, we may again make use of the superfast accelerated projected gradient method using the gradient operator $\delta \mathscr{D} / \delta \rho^{\prime}$ for the minENT procedure instead of $\delta \ln L / \delta \rho^{\prime}$ for the usual ML optimization considered in [47]. For this purpose, we supply a simple instruction manual to modify and use the open-source MATLAB code file qse_apg.m that is available at [50]. The three important variables are fval_varrho, fval_new, and gradient, which stores the function values of $\mathscr{D}$ evaluated with the varrho and rho_new variables, and the gradient operator

$$
\frac{\delta \mathscr{D}}{\delta \rho^{\prime}}=\lambda\left(1+\ln \rho^{\prime}\right)+\sum_{j^{\prime}, l^{\prime}, k^{\prime}}\left|b_{l^{\prime} k^{\prime}}^{\left(j^{\prime}\right)}\right\rangle \frac{v_{l^{\prime} k^{\prime}}^{\left(j^{\prime}\right)}}{\left\langle b_{l^{\prime} k^{\prime}}^{\left(j^{\prime}\right)}\left|\rho^{\prime}\right| b_{l^{\prime} k^{\prime}}^{\left(j^{\prime}\right)}\right.}\left\langle b_{l^{\prime} k^{\prime}}^{\left(j^{\prime}\right)}\right|
$$

evaluated with varrho. In order to minimize $\mathscr{D}$ using qse_apg.m, we may simply overwrite the existing functional expressions [namely $-f^{\prime} \cdot * \log \left(\operatorname{probs}_{-} \ldots\right)$ and $-\operatorname{qmt}(\ldots)]$ for the three variables with those in Eqs. (A1) and (2).

\section{EXPLICIT PROCEDURES}

We shall state the operational protocol of (P)ACTQPT pictorialized in Fig. 1.
[(P)ACTQPT] At the $j$ th step.

(1) All measured bases $\left\{\mathscr{B}^{(1)}, \ldots, \mathscr{B}^{(j)}\right\}$, ML probabilities $\left\{\widehat{\boldsymbol{p}}^{(1)}, \ldots, \widehat{\boldsymbol{p}}^{(j)}\right\}$, and estimates of relative trace values $\left\{\widehat{\mu}_{1}, \ldots, \widehat{\mu}_{j}\right\}$ of the output states, all obtained from (P)ACT, are first used to compute the LS value $\mathscr{D}_{\text {min }}$ defined in Eq. (1) over the space of quantum processes.

(2) The resulting LS (non-TP) probabilities $\left\{\widehat{\boldsymbol{p}}_{\mathrm{LS}}^{(1)}, \ldots, \widehat{\boldsymbol{p}}_{\mathrm{LS}}^{(j)}\right\}$ that correspond to $\mathscr{D}_{\min }$, together with the measured bases, are next subject to ICC, which are two SDPs that lead to $s_{\mathrm{CVX}, j}^{(\Phi)}$ by minimizing and maximizing $f=\operatorname{tr}\left\{\rho_{\Phi}^{\prime} Z\right\}$ for some fixed $d^{2}$ dimensional full-rank state $Z$ over the LS convex set $\mathscr{C}_{j}^{(\Phi)}$ that is defined by (a) the complete-positivity quantum constraint $\rho_{\Phi}^{\prime} \geqslant 0$, (b) the linear LS constraints $\left\langle b_{l^{\prime} k^{\prime}}^{\left(j^{\prime}\right)} \widehat{\Phi}\left[\rho_{\mathrm{IN}}^{\left(j^{\prime}\right)}\right] \mid b_{l^{\prime} k^{\prime}}^{\left(j^{\prime}\right)}\right\rangle=$ $\widehat{p}_{\mathrm{LS}, l^{\prime} k^{\prime}}^{\left(j^{\prime}\right)}$ for $0 \leqslant l^{\prime} \leqslant d-1,1 \leqslant k^{\prime} \leqslant K_{j^{\prime}}$, and $1 \leqslant j^{\prime} \leqslant j$, and (c) the trace constraint $\operatorname{tr}\left\{\rho_{\Phi}^{\prime}\right\}=\operatorname{tr}\left\{\hat{\rho}_{\Phi}^{(\mathrm{LS})}\right\}$ for a particular LS process estimator $\widehat{\rho}_{\Phi}^{(\mathrm{LS})} \in \mathscr{C}_{j}^{(\Phi)}$, where this final constraint mainly serves to stabilize the incorporation of the LS constraints.

(3) (P)ACTQPT terminates when $s_{\mathrm{CVX}, j}^{(\Phi)}$ is less than some threshold value.

(4) Otherwise, it proceeds to carry out (P)ACT for a new randomly chosen input state, and $j$ is raised by one.

The (P)ACT subprotocol is yet another self-consistent scheme that reconstructs any quantum state through adaptive compression. After $\rho_{\mathrm{IN}}^{(j)}$ is evolved by the unknown $\Phi$, the corresponding unknown $\rho_{\text {OUT }}^{(j)}$ undergoes an adaptive compressive iterative run.

[(P)ACT] Starting with $k=1$.

(1) The scheme measures the basis $\mathscr{B}_{k}$ and collects the relative frequency data $\sum_{l^{\prime}=0}^{d-1} v_{l^{\prime} k}^{(j)}=1$.

(2) From the accumulated data set $\left\{v_{0 k^{\prime}}^{(j)}, \ldots, v_{d-1}^{(j)}{ }_{k^{\prime}}\right\}_{k^{\prime}=1}^{k}$, the corresponding physical ML probabilities $\left\{\widehat{p}_{0 k^{\prime}}^{(j)}\right.$, $\left.\ldots, \widehat{p}_{d-1 k^{\prime}}^{(j)}\right\}_{k^{\prime}=1}^{k}$ are computed according to Appendix A.

(3) They are then used to perform ICC, where the uniqueness indicator $s_{\mathrm{CVX}, k}^{(\rho)}$ is obtained by running two SDPs that find the unique minimum and maximum values of $f=\operatorname{tr}\left\{\rho^{\prime} Y\right\}$, with a fixed $d$-dimensional full-rank state $Y$, over the ML convex set $\mathscr{C}_{k}^{(\rho)}$, which is defined by (a) the positivity quantum constraint $\rho^{\prime} \geqslant 0$, (b) unit-trace constraint $\operatorname{tr}\left\{\rho^{\prime}\right\}=1$, and (c) the linear ML constraints $\left\langle b_{l^{\prime} k^{\prime}}^{(j)}\left|\rho^{\prime}\right| b_{l^{\prime} k^{\prime}}^{(j)}\right\rangle=\widehat{p}_{l^{\prime} k^{\prime}}^{(j)}$ for $0 \leqslant l^{\prime} \leqslant$ $d-1$ and $1 \leqslant k^{\prime} \leqslant k$.

(4) (P)ACT terminates if $s_{\mathrm{CVX}, k}$ is less than some prechosen threshold. Otherwise, it proceeds to choose the next optimal basis $\mathscr{B}_{k+1}$ to measure in the $(k+1)$ th step.

(5) The basis $\mathscr{B}_{k+1}$ is chosen adaptively by first finding the minENT estimator over $\mathscr{C}_{k}^{(\rho)}$. From here, ACT directly takes $\mathscr{B}_{k+1}^{(j)}$ to be the eigenbasis of the minENT estimator, whereas PACT defines $\mathscr{B}_{k+1}^{(j)}$ as the nearest tensor-product basis to this optimal eigenbasis, say, according to $\left\|U_{\text {prod }}-U\right\|_{2}$, the 2-norm distance between the product unitary $U_{\text {prod }}$ acting on some reference product basis and optimal unitary $U$ that rotates the reference product basis to the eigenbasis of the $\operatorname{minENT} \widehat{\rho}_{k}$.

(6) Then, $k$ is raised by one and (P)ACT repeats itself until $s_{\mathrm{CVX}, k}^{(\rho)} \approx 0$. 


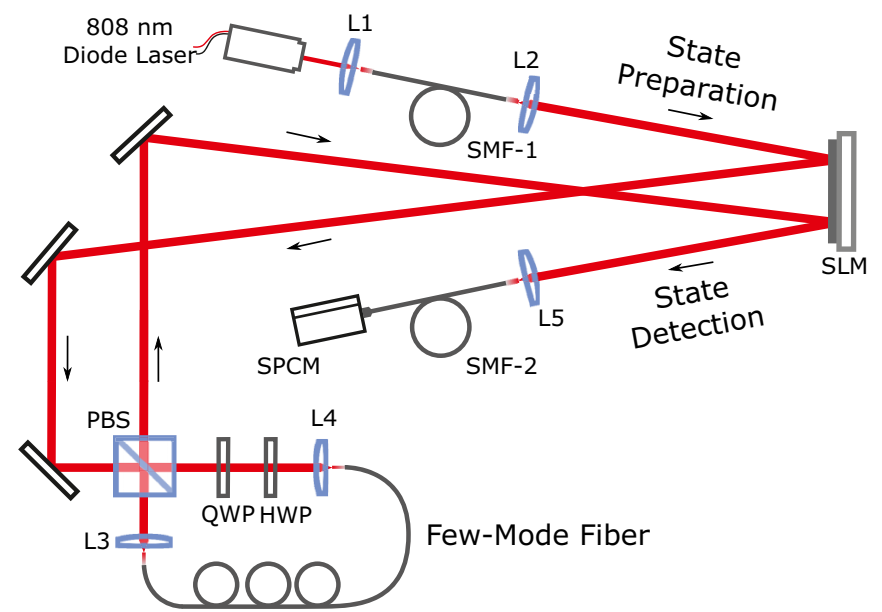

FIG. 2. Experimental setup for preparation and measurement of spatial-qudit states for rank-deficient quantum processes occurring in a few-mode fiber. State preparation was realized with a hologram displayed on the top half of the SLM. A subsequent state detection was performed by the hologram on the SLM's bottom half followed by a single-mode fiber (SMF-2) and a single-photon counting module (SPCM).

\section{NUMERICAL AND EXPERIMENTAL RESULTS AND ANALYSIS}

The figure of merit for analyzing the performances of (P)ACTQPT is the IC number of measurement configurations $\left(M_{\mathrm{IC}}\right)$ needed to unambiguously reconstruct $\Phi$, which is the grand total of output-state measurement outcomes in a full (P)ACTQPT run. We experimentally tested (P)ACTQPT using transverse modes of light to flexibly emulate complex quantum systems of various dimensions $d$. The corresponding infinite-dimensional Hilbert space is now represented in the Hermite-Gaussian basis. Attenuated light from a diode laser of $808 \mathrm{~nm}$ wavelength was filtered by a single-mode fiber (SMF1) and then collimated by an aspheric lens L2 (see Fig. 2). The top half of the spatial light modulator (SLM) (Holoeye Pluto) generates the desired spatial state of the photon, and its bottom half, followed by a single-mode fiber (SMF-2), implements projective measurements of the transformed state as in [51,52]. To precalibrate the setup, we performed preliminary (P)ACTQPT runs with the idle process, the results of which are in Appendix B.

In Fig. 3, we compare (P)ACTQPT with a proposed optimal benchmark provided by the Baldwin-Kalev-Deutsch (BKD) scheme [37], requiring both the unitarity assumption and $M_{\mathrm{BKD}}=d^{2}+d$ nonprojective entangling measurements. Few-mode optical fibers, which behave as rank-deficient processes acting on two-qubit $(d=4)$ and two-qutrit systems $(d=9)$, are characterized with (P)ACTQPT. To realize these processes, we coupled the photons after the polarizing beam splitter (PBS) into a one-meter-long few-mode optical fiber. The utilized fiber (SMF-28) supported the propagation of HG modes of four lowest orders at the $808 \mathrm{~nm}$ operating wavelength, which is red detuned from the designed wavelength of $1.5 \mu \mathrm{m}$ for the single-mode regime. We were able to control the output mode content by altering input polarization using the half-wave (HWP) and quarter-wave (QWP) plates, followed by the PBS. This is possible due to cross-coupling between polarization and spatial degrees of freedom in optical fibers [53].

In all of the experiments, the basis projectors are measured sequentially, one at a time, by maintaining a particular measurement configuration for some fixed duration. Hence, for the noisy simulations, noise on each basis projector $\left|b_{l^{\prime} k^{\prime}}^{\left(j^{\prime}\right)}\right\rangle\left\langle b_{l^{\prime} k^{\prime}}^{\left(j^{\prime}\right)}\right|$ is modeled with a single-variable Gaussian distribution, with

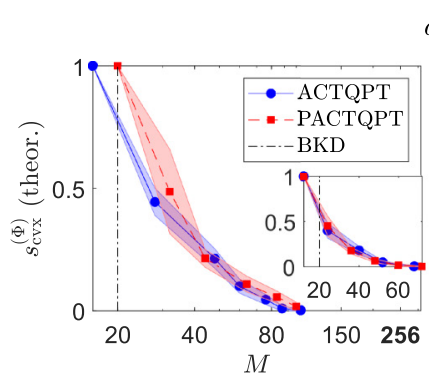

$d=4$
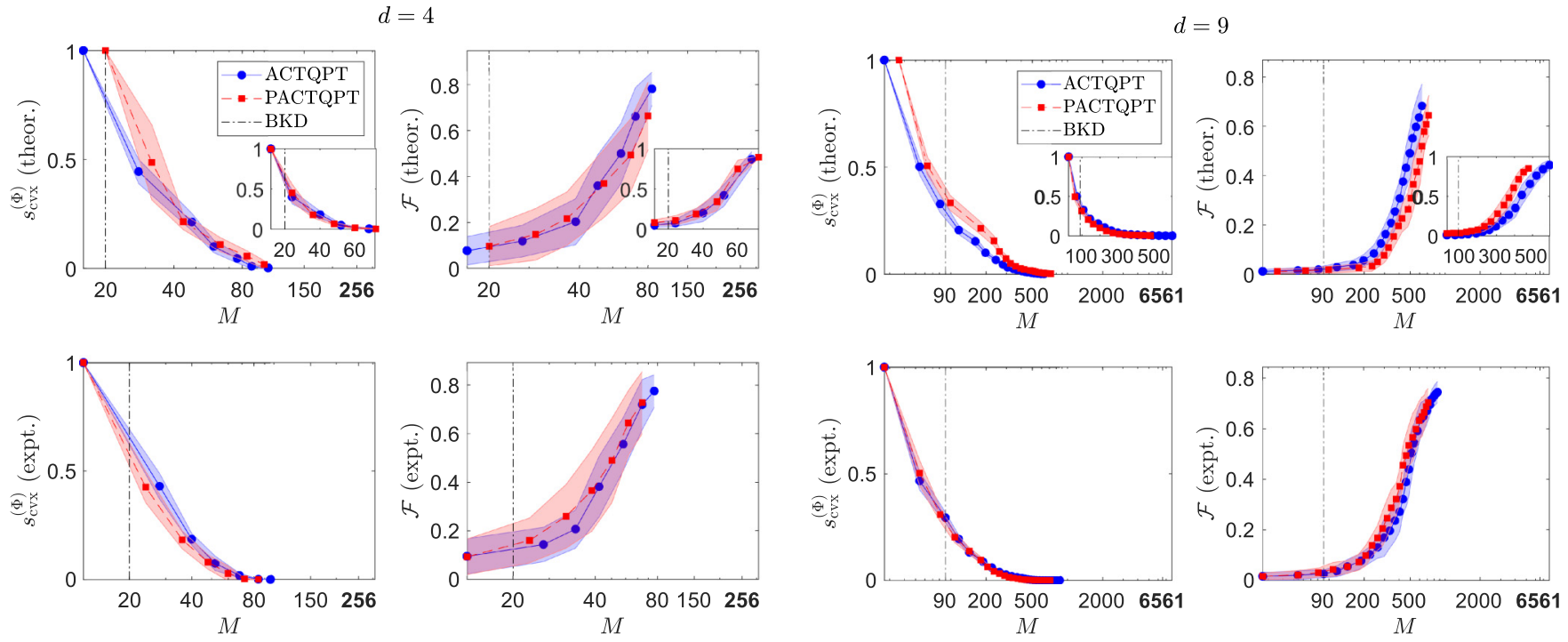

FIG. 3. Numerical and experimental plots and 1- $\sigma$ error regions of both $s_{\mathrm{CVx}}^{(\Phi)}$ and $\mathscr{F}$ for rank-4 two-qubit $(d=4)$ and rank-10 two-qutrit processes $(d=9)$ against $M$, averaged over 40 and 20 runs, respectively. Explicitly, the $d=4$ process operator has four positive eigenvalues $0.8721,0.1062,0.0160$, and 0.0057 , while the $d=9$ process operator has 10 positive eigenvalues $0.9253,0.0252,0.0200,0.0125,0.0082$, $0.0043,0.0025,0.0014,0.0005$, and 0.0002 . Numerical results with noiseless data are shown in the insets, whereas simulations with noisy data $(\eta=0.01$ and 0.005 , respectively, for the two processes) explain the actual experiments very well. The results are benchmarked with the optimal BKD schemes of $M_{\mathrm{BKD}}=4^{2}+4=20$ and $9^{2}+9=90$. The $s_{\mathrm{CVX}}$ threshold is set to $10^{-3}$. 
TABLE I. Average $M_{\text {IC }}$ values and their standard deviations for the two-qubit and two-qutrit fiber processes studied in Fig. 3.

\begin{tabular}{|c|c|c|c|c|}
\hline & & Theor. $(\eta=0)$ & Theor. $(\eta=0.01)$ & Expt. \\
\hline \multirow{3}{*}{$\begin{array}{l}\mathcal{f} \\
\| \\
\overparen{J}\end{array}$} & ACTQPT & $89 \pm 10$ & $126 \pm 16$ & $115 \pm 12$ \\
\hline & PACTQPT & $97 \pm 12$ & $133 \pm 21$ & $123 \pm 16$ \\
\hline & & Theor. $(\eta=0)$ & Theor. $(\eta=0.005)$ & Expt. \\
\hline \multirow{2}{*}{$\begin{array}{l}\widehat{\sigma} \\
\stackrel{\theta}{\Xi}\end{array}$} & ACTQPT & $621 \pm 56$ & $885 \pm 109$ & $927 \pm 25$ \\
\hline & PACTQPT & $613 \pm 37$ & $864 \pm 100$ & $809 \pm 46$ \\
\hline
\end{tabular}

mean $p_{l^{\prime} k^{\prime}}^{\left(j^{\prime}\right)}$ and variance set to an effective value $\eta^{2}$ that is common to all projectors. The value of $\eta>0$ is chosen to match the experimental results in order to explain the observed noise with an effective Gaussian noise model. These simulations assume the true processes $\rho_{\Phi} \equiv \widehat{\rho}_{\Phi}^{\text {(overcomp) }}$ determined via overcomplete tomography.

Noiseless simulation results of rank-1 processes, each taken as the largest-eigenvalue projector of the corresponding $\widehat{\rho}_{\Phi}^{\text {(overcomp) }}$, are presented for idealized comparisons. The fidelity $\mathscr{F}=\operatorname{tr}\left\{\sqrt{\widehat{\rho}_{\Phi}} \rho_{\Phi} \sqrt{\widehat{\rho}_{\Phi}}\right\} /\left(\operatorname{tr}\left\{\widehat{\rho}_{\Phi}\right\} \operatorname{tr}\left\{\rho_{\Phi}\right\}\right)$ is defined between two general non-TP process operators. For these simulations, we assume the ideal situation where perfect fibers are used. These implies that the resulting quantum-process operators are all rank-one.

The average $M_{\mathrm{IC}}$ values of (P)ACTQPT for Fig. 3 are presented in Table I, where we find that there is not a very big difference in the average $M_{\mathrm{IC}}$ relative to $O\left(d^{4}\right)$ between ACTQPT and PACTQPT for the tested processes. Furthermore, different choices of optimal adaptive bases and confounding experimental factors can result in varying relative performances. We attribute their performance similarity to the inherent input-output characteristic framework of these QPT schemes, which can limit further enhancements with entangled input states and basis measurements. Our results also demonstrate that (P)ACTQPT is highly compressive. Both the two-qubit and two-qutrit experiments respectively show over $50 \%$ and $85 \%$ reduction in IC measurement resources relative to $O\left(d^{4}\right)$ without spurious prior assumptions of any sort about the processes. From the table and figures, if one takes the rank

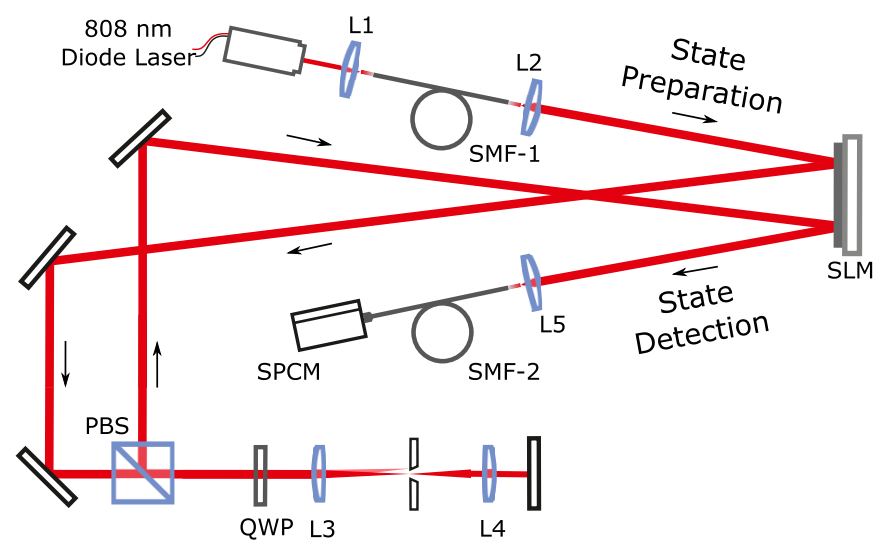

FIG. 4. Schematic diagram of the setup that implements ACTQPT for the idle process.

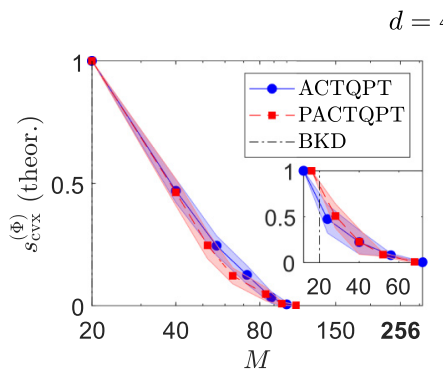

$$
d=4 \text { (idle) }
$$
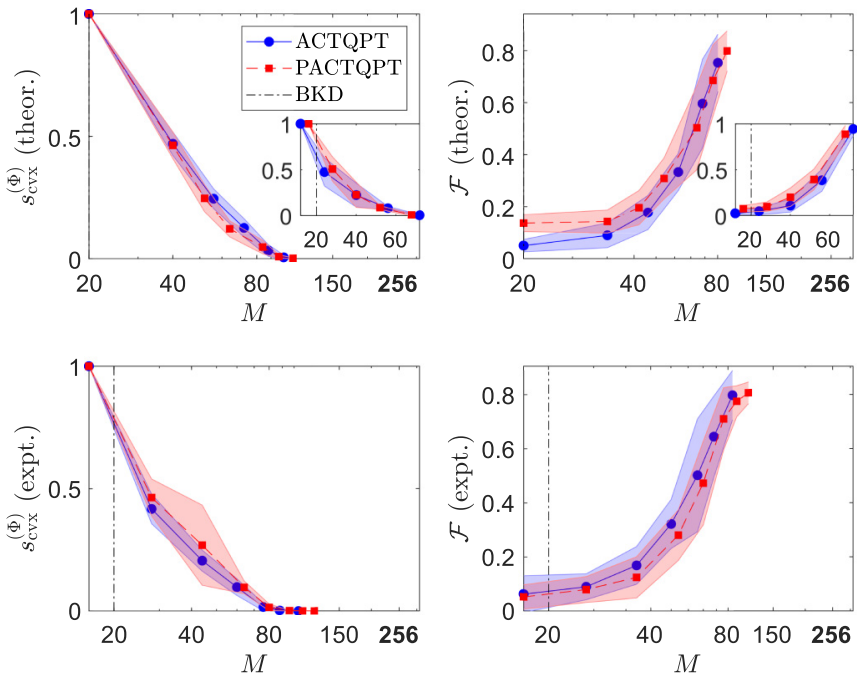

FIG. 5. Numerical and experimental plots and 1- $\sigma$ error regions of both $s_{\mathrm{CVX}}^{(\Phi)}$ and $\mathscr{F}$ for the two-qubit idle process $(d=4)$ against $M$, averaged over 40 and 20 runs, respectively. Numerical results with noiseless data are shown in the insets, whereas simulations with noisy data $(\eta=0.01)$ explain the actual experiments very well. The results are compared with the optimal benchmark $\left(M_{\mathrm{BKD}}=20\right)$. The $s_{\mathrm{CVX}}$ threshold is set to $10^{-3}$.

of an unknown process for granted, one presumably gets away with a further $\approx 90 \%$ reduction in measurement resources that are, especially in our case, of paramount importance for validating this rank assumption and final reconstruction answer.

\section{CONCLUSION}

We have successfully demonstrated a compressive statereconstruction-assisted quantum process tomography method that requires no a priori assumptions about the unknown quantum process, in particular not the frequently taken-forgranted rank or sparsity assumption, to fully characterize the process with much fewer measurement resources than the fourth power of the system dimension. It involves a uniqueness reconstruction certification procedure over the general completely positive quantum-process space and a recently established adaptive compressive state tomography scheme. Our results with experimentally implemented processes indicate that the compressive method works equally well with both entangled and product input states and output measurement resources even in the presence of noise. This allows one to implement this method in practice using uncorrelated quantum resources without precise and expensive global entangling operations.

\section{ACKNOWLEDGMENTS}

We acknowledge financial support from the BK21 Plus Program (No. 21A20131111123) funded by the Ministry of Education (MOE, Korea) and National Research Foundation of Korea (NRF), the NRF grant funded by the Korea government (MSIP) (Grants No. NRF-2019M3E4A1080074, 


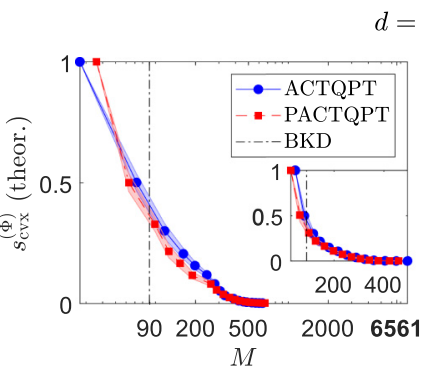

$d=9($ idle $)$
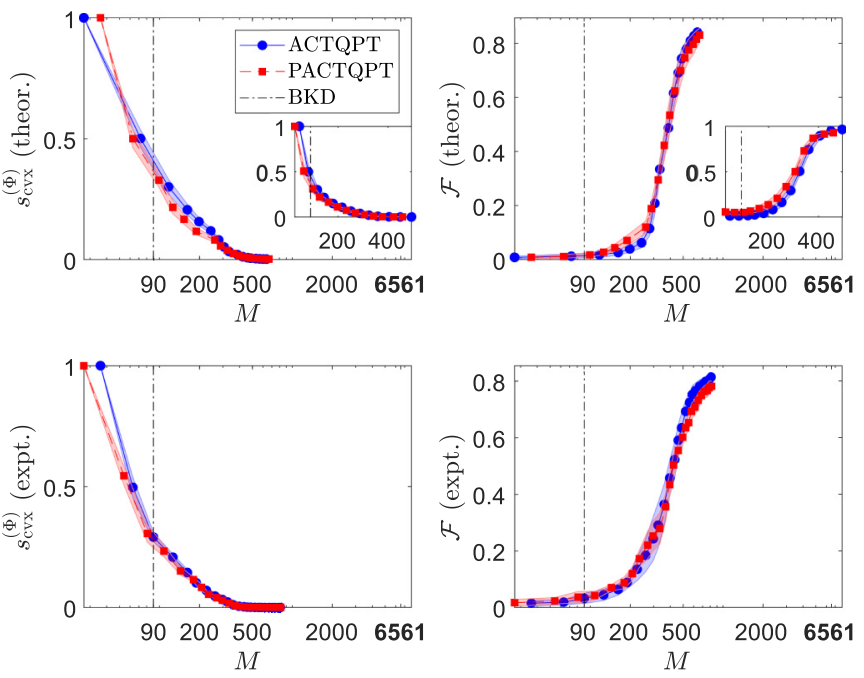

FIG. 6. Numerical and experimental plots and 1- $\sigma$ error regions of both $s_{\mathrm{CVX}}^{(\Phi)}$ and $\mathscr{F}$ for the two-qutrit idle process $(d=9)$ against $M$, averaged over 40 and 10 runs, respectively. Here, $\eta=0.00375$ for noisy simulations. The optimal BKD benchmark here is $M_{\mathrm{BKD}}=90$, and the average $M_{\mathrm{IC}}$ 's for both ACTQPT and PACTQPT are again comparable and about an order of magnitude smaller than $d^{4}=$ 6561. All other figure specifications are otherwise the same as those of Fig. 5.

No. NRF-2019R1H1A3079890, and No. NRF2018K2A9A1A06069933), Russian Foundation for Basic Research (RFBR Projects No. 19-32-80043 and No. 19-52-80034), Mega-grant of the Ministry of Education and Science of the Russian Federation (Contract No. 14.W03.31.0032), and the Spanish MINECO (Grants No. FIS2015-67963-P and No. PGC2018-099183-B-I00).

\section{APPENDIX A: MAXIMUM-LIKELIHOOD PHYSICAL PROBABILITIES}

The normalized frequency data $\mathbb{D}=\left\{v_{l^{\prime} k^{\prime}}^{\left(j^{\prime}\right)}\right\}$ collected in ACT are noisy and, almost always, do not correspond to any physical quantum state. We therefore need to estimate the correct physical probabilities $\widehat{p}_{l^{\prime} k^{\prime}}^{\left(j^{\prime}\right)}$ that asymptotically approach the true ones in the limit of large sampling copies.

A good statistical approach for dealing with a finite number of sampling events is to first identify the likelihood function $L\left(\mathbb{D} \mid \rho^{\prime}\right)$ for the experiment, which takes the multinomial form in the situation where the total number of registered counts $N$ is fixed and every measurement count is independent. The corresponding concave multinomial (normalized) log-likelihood
TABLE II. Average $M_{\mathrm{IC}}$ values and their standard deviations for the two-qubit and two-qutrit idle processes in Figs. 5 and 6.

\begin{tabular}{ccccc}
\hline \hline & & Theor. $(\eta=0)$ & Theor. $(\eta=0.01)$ & Expt. \\
\hline \multirow{f}{*}{} & ACTQPT & $83 \pm 9$ & $116 \pm 15$ & $118 \pm 11$ \\
& PACTQPT & $95 \pm 13$ & $131 \pm 14$ & $140 \pm 16$ \\
& & Theor. $(\eta=0)$ & Theor. $(\eta=0.00375)$ & Expt. \\
$\overparen{\sigma}$ & ACTQPT & $496 \pm 28$ & $871 \pm 70$ & $880 \pm 65$ \\
II & PACTQPT & $502 \pm 36$ & $847 \pm 88$ & $838 \pm 27$ \\
\hline \hline
\end{tabular}

expression reads

$$
\ln L\left(\mathbb{D} \mid \rho^{\prime}\right)=\sum_{j^{\prime}, l^{\prime}, k^{\prime}} v_{l^{\prime} k^{\prime}}^{\left(j^{\prime}\right)} \ln \left\langle b_{l^{\prime} k^{\prime}}^{\left(j^{\prime}\right)}\left|\rho^{\prime}\right| b_{l^{\prime} k^{\prime}}^{\left(j^{\prime}\right)}\right\rangle .
$$

This likelihood function has the meaning of a conditional probability of obtaining $\mathbb{D}$ given the state $\rho^{\prime}$, and maximizing this function over all quantum states shall then give the mostlikely physical state $\widehat{\rho} \geqslant 0$ that gives $\mathbb{D}$. The estimated ML physical probabilities are then $\widehat{p}_{l^{\prime} k^{\prime}}^{\left(j^{\prime}\right)}=\left\langle b_{l^{\prime} k^{\prime}}^{\left(j^{\prime}\right)}|\widehat{\rho}| b_{l^{\prime} k^{\prime}}^{\left(j^{\prime}\right)}\right\rangle$.

One may adopt the steepest-ascent algorithm [44-46] to maximize $L$. The most efficient algorithm to date, however, can be derived according to the accelerated projected-gradient recipe, where at its core is an augmented rapid-converging iteration of a likelihood maximization over all unit-trace Hermitian operators followed by a projection onto the unit-trace positive-operator space [47]. Without restating the entire code, we refer the reader to the GitHub page [50] for ready-to-use MATLAB codes, supplemented with tutorials and examples.

\section{APPENDIX B: EXPERIMENTAL PRECALIBRATION WITH THE IDLE PROCESS}

Before the actual experiments with few-mode fibers, the performance of the setup was first evaluated with the control idle process. In the ideal situation, this corresponds to the trivial map $\Phi: \rho \mapsto \rho$ for every quantum state $\rho$. In real experiments, however, the presence of Gouy phases [52] leads to the map $\Phi: \rho \mapsto U_{\text {idle }} \rho U_{\text {idle }}^{\dagger}$, where $U_{\text {idle }}$ is a diagonal unitary operator. In our setup shown in Fig. 4, the idle process is implemented by using the combination of lenses L 3 and L4 with equal focal lengths $(100 \mathrm{~mm})$ separated by a $200 \mathrm{~mm}$ distance. Since the holograms displayed on the SLM were based on the blazed grating [54], the pinhole in the focal plane was used for the state selection in the first diffraction order. After the double pass through the telescope and a quarterwave plate (QWP) the beam was reflected by the PBS and directed back to the SLM without any additional alterations. All simulation and experimental results are showcased in Figs. 5 and 6, with the precise $M_{\text {IC }}$ listed in Table II.
[1] T. D. Ladd, F. Jelezko, R. Laflamme, Y. Nakamura, C. Monroe, and J. L. O'Brien, Quantum computers, Nature (London) 464, 45 (2010).

[2] E. T. Campbell, B. M. Terhal, and C. Vuillot, Roads towards fault-tolerant universal quantum computation, Nature (London) 549, 172 (2017).
[3] B. Lekitsch, S. Weidt, A. G. Fowler, K. Mølmer, S. J. Devitt, Ch. Wunderlich, and W. K. Hensinger, Blueprint for a microwave trapped ion quantum computer, Sci. Adv. 3, e1601540 (2017).

[4] V. M. Schäfer, C. J. Ballance, K. Thirumalai, L. J. Stephenson, T. G. Ballance, A. M. Steane, and D. M. Lucas, Fast quantum 
logic gates with trapped-ion qubits, Nature (London) 555, 75 (2018).

[5] X.-F. Shi, Accurate Quantum Logic Gates by Spin Echo in Rydberg Atoms, Phys. Rev. Appl. 10, 034006 (2018).

[6] T. Ono, R. Okamoto, M. Tanida, H. F. Hofmann, and S. Takeuchi, Implementation of a quantum controlled-swap gate with photonic circuits, Sci. Rep. 7, 45353 (2017).

[7] R. B. Patel, J. Ho, F. Ferreyrol, T. C. Ralph, and G. J. Pryde, A quantum Fredkin gate, Sci. Adv. 2, e1501531 (2016).

[8] J. Fiurášek, Linear optical Fredkin gate based on partial-swap gate, Phys. Rev. A 78, 032317 (2008).

[9] M. Choi, Completely positive linear maps on complex matrices, Linear Alg. Appl. 10, 285 (1975).

[10] A. Jamiołkowski, Linear transformations which preserve trace and positive semidefiniteness of operators, Rep. Math. Phys. 3, 275 (1972).

[11] I. Chuang and M. Nielsen, Quantum Computation and Quantum Information (Cambridge University Press, Cambridge, UK, 2000).

[12] J. L. O’Brien, G. J. Pryde, A. Gilchrist, D. F. V. James, N. K. Langford, T. C. Ralph, and A. G. White, Quantum Process Tomography of a Controlled-Not Gate, Phys. Rev. Lett. 93, 080502 (2004).

[13] J. Fiurášek, Maximum-likelihood estimation of quantum measurement, Phys. Rev. A 64, 024102 (2001).

[14] J. F. Poyatos, J. I. Cirac, and P. Zoller, Complete Characterization of a Quantum Process: The Two-Bit Quantum Gate, Phys. Rev. Lett. 78, 390 (1997).

[15] J. B. Altepeter, D. Branning, E. Jeffrey, T. C. Wei, P. G. Kwiat, R. T. Thew, J. L. O'Brien, M. A. Nielsen, and A. G. White, Ancilla-Assisted Quantum Process Tomography, Phys. Rev. Lett. 90, 193601 (2003).

[16] D. W. Leung, Choi's proof as a recipe for quantum process tomography, J. Math. Phys. 44, 528 (2003).

[17] G. M. D'Ariano and P. Lo Presti, Imprinting Complete Information About a Quantum Channel on its Output State, Phys. Rev. Lett. 91, 047902 (2003).

[18] G. M. D'Ariano and P. Lo Presti, Quantum Tomography for Measuring Experimentally the Matrix Elements of an Arbitrary Quantum Operation, Phys. Rev. Lett. 86, 4195 (2001).

[19] S. Omkar, R. Srikanth, and S. Banerjee, Characterization of quantum dynamics using quantum error correction, Phys. Rev. A 91, 012324 (2015).

[20] S. Omkar, R. Srikanth, and S. Banerjee, Quantum code for quantum error characterization, Phys. Rev. A 91, 052309 (2015).

[21] M. Mohseni and D. A. Lidar, Direct characterization of quantum dynamics: General theory, Phys. Rev. A 75, 062331 (2007).

[22] M. Mohseni and D. A. Lidar, Direct Characterization of Quantum Dynamics, Phys. Rev. Lett. 97, 170501 (2006).

[23] Y. Kim, Y.-S. Kim, S.-Y. Lee, S.-W. Han, S. Moon, Y.-H. Kim, and Y.-W. Cho, Direct quantum process tomography via measuring sequential weak values of incompatible observables, Nat. Commun. 9, 192 (2018).

[24] A. Gaikwad, D. Rehal, A. Singh, Arvind, and K. Dorai, Experimental demonstration of selective quantum process tomography on an NMR quantum information processor, Phys. Rev. A 97, 022311 (2018).
[25] A. Bendersky and J. P. Paz, Selective and efficient quantum state tomography and its application to quantum process tomography, Phys. Rev. A 87, 012122 (2013).

[26] C. T. Schmiegelow, A. Bendersky, M. A. Larotonda, and J. P. Paz, Selective and Efficient Quantum Process Tomography without Ancilla, Phys. Rev. Lett. 107, 100502 (2011).

[27] A. Bendersky, F. Pastawski, and J. P. Paz, Selective and efficient quantum process tomography, Phys. Rev. A 80, 032116 (2009).

[28] A. Bendersky, F. Pastawski, and J. P. Paz, Selective and Efficient Estimation of Parameters for Quantum Process Tomography, Phys. Rev. Lett. 100, 190403 (2008).

[29] Y. S. Teo, B.-G. Englert, J. Řeháček, and Z. Hradil, Adaptive schemes for incomplete quantum process tomography, Phys. Rev. A 84, 062125 (2011).

[30] D. Donoho, Compressed sensing, IEEE Trans. Inf. Theory 52, 1289 (2006).

[31] E. J. Candès and T. Tao, Near-optimal signal recovery from random projections: Universal encoding strategies? IEEE Trans. Inf. Theory 52, 5406 (2006).

[32] E. J. Candès and B. Recht, Exact matrix completion via convex optimization, Found. Comput. Math. 9, 717 (2009).

[33] D. Gross, Y.-K. Liu, S. T. Flammia, S. Becker, and J. Eisert, Quantum State Tomography via Compressed Sensing, Phys. Rev. Lett. 105, 150401 (2010).

[34] A. Kalev, R. L. Kosut, and I. H. Deutsch, Quantum tomography protocols with positivity are compressed sensing protocols, npj Quantum Inf. 1, 15018 (2015).

[35] A. Steffens, C. A. Riofrío, W. McCutcheon, I. Roth, B. A. Bell, A. McMillan, M. S. Tame, J. G. Rarity, and J. Eisert, Experimentally exploring compressed sensing quantum tomography, Quantum Sci. Technol. 2, 025005 (2017).

[36] C. A. Riofrío, D. Gross, S. T. Flammia, T. Monz, D. Nigg, R. Blatt, and J. Eisert, Experimental quantum compressed sensing for a seven-qubit system, Nat. Commun. 8, 15305 (2017).

[37] C. H. Baldwin, A. Kalev, and I. H. Deutsch, Quantum process tomography of unitary and near-unitary maps, Phys. Rev. A 90, 012110 (2014).

[38] A. V. Rodionov, A. Veitia, R. Barends, J. Kelly, D. Sank, J. Wenner, J. M. Martinis, R. L. Kosut, and A. N. Korotkov, Compressed sensing quantum process tomography for superconducting quantum gates, Phys. Rev. B 90, 144504 (2014).

[39] A. Shabani, R. L. Kosut, M. Mohseni, H. Rabitz, M. A. Broome, M. P. Almeida, A. Fedrizzi, and A. G. White, Efficient Measurement of Quantum Dynamics via Compressive Sensing, Phys. Rev. Lett. 106, 100401 (2011).

[40] D. Ahn, Y. S. Teo, H. Jeong, F. Bouchard, F. Hufnagel, E. Karimi, D. Koutný, J. Řeháček, Z. Hradil, G. Leuchs, and L. L. Sánchez-Soto, Adaptive Compressive Tomography with No a priori Information, Phys. Rev. Lett. 122, 100404 (2019).

[41] D. Ahn, Y. S. Teo, H. Jeong, D. Koutný, J. Řeháček, Z. Hradil, G. Leuchs, and L. L. Sánchez-Soto, Adaptive compressive tomography: A numerical study, Phys. Rev. A 100, 012346 (2019).

[42] L. Vandenberghe and S. Boyd, Semidefinite programming, SIAM Rev. 38, 49 (1996).

[43] S. Boyd and L. Vandenberghe, Convex Optimization (Cambridge University Press, Cambridge, UK, 2004).

[44] J. Řeháček, Z. Hradil, E. Knill, and A. I. Lvovsky, Diluted maximum-likelihood algorithm for quantum tomography, Phys. Rev. A 75, 042108 (2007). 
[45] Y. S. Teo, H. Zhu, B.-G. Englert, J. Řeháček, and Z. Hradil, Quantum-State Reconstruction by Maximizing Likelihood and Entropy, Phys. Rev. Lett. 107, 020404 (2011).

[46] Y. S. Teo, Introduction to Quantum-State Estimation (World Scientific Publishing Co., Singapore, 2015).

[47] J. Shang, Z. Zhang, and H. K. Ng, Superfast maximumlikelihood reconstruction for quantum tomography, Phys. Rev. A 95, 062336 (2017).

[48] S. Huang, D. N. Tran, and T. D. Tran, Sparse signal recovery based on nonconvex entropy minimization, in 2016 IEEE International Conference on Image Processing (ICIP) (IEEE, New York, 2016), p. 3867.

[49] D. N. Tran, S. Huang, S. P. Chin, and T. D. Tran, Low-rank matrices recovery via entropy function, in 2016 IEEE International Conference on Acoustics, Speech and Signal Processing (ICASSP) (IEEE, New York, 2016), p. 4064.
[50] https://github.com/qMLE/qMLE.

[51] N. Bent, H. Qassim, A. A. Tahir, D. Sych, G. Leuchs, L. L. Sánchez-Soto, E. Karimi, and R. W. Boyd, Experimental Realization of Quantum Tomography of Photonic Qudits via Symmetric Informationally Complete Positive Operator-Valued Measures, Phys. Rev. X 5, 041006 (2015).

[52] A. Macarone-Palmieri, E. Kovlakov, F. Bianchi, D. Yudin, S. Straupe, J. Biamonte, and S. Kulik, Experimental neural network enhanced quantum tomography, npj Quantum Inf. 6, 20 (2020).

[53] J. Carpenter, C. Xiong, M. J. Collins, J. Li, T. F. Krauss, B. J. Eggleton, A. S. Clark, and J. Schröder, Mode multiplexed single-photon and classical channels in a few-mode fiber, Opt. Express 21, 28794 (2013).

[54] E. Bolduc, N. Bent, E. Santamato, E. Karimi, and R. W. Boyd, Exact solution to simultaneous intensity and phase encryption with a single phase-only hologram, Opt. Lett. 38, 3546 (2013). 